\title{
RANDOM ENTROPY AND RECURRENCE
}

\author{
KARMA DAJANI and RONALD MEESTER
}

Received 19 December 2002

\begin{abstract}
We show that a cocycle, which is nothing but a generalized random walk with index set $\mathbb{Z}^{d}$, with bounded step sizes is recurrent whenever its associated random entropy is zero, and transient whenever its associated random entropy is positive. This generalizes a well-known one-dimensional result and implies a Polya type dichotomy for this situation.
\end{abstract}

2000 Mathematics Subject Classification: 37A50, 28D20.

1. Motivation and introduction. In [1], the concept of random entropy associated with a $\mathbb{Z}^{d}$ random group action was introduced and studied. Every such $\mathbb{Z}^{d}$ random group action is generated via a cocycle. (For the readers with a probabilistic background, a cocycle is a generalization of an ordinary random walk, the main difference being the fact that cocycles are generally indexed by $\mathbb{Z}^{d}$ rather than by $\mathbb{Z}$. A one-dimensional cocycle is nothing but an ordinary random walk; we give precise definitions in Section 2.) In the one-dimensional case, it is easy to see that having positive random entropy is equivalent to the transience of the associated random walk. It therefore seems reasonable to try to connect the concept of random entropy as developed in [1] and the transience of the generating cocycle. In this paper, we show that the one-dimensional connection holds in general.

The paper is completely self-contained. Section 2 contains the setup, including all necessary definitions and the main results, while Sections 3 and 4 contain the proofs.

2. Cocycles and random entropy. Let $\Omega$ be the following set:

$$
\begin{aligned}
\Omega=\{\omega= & \left(\left(\omega_{z}^{1}, \ldots, \omega_{z}^{d}\right)_{z \in \mathbb{Z}^{d}}\right) ; \omega_{z}^{i} \in \mathbb{Z}^{d}, \\
& \left.\omega_{z}^{i}+\omega_{z+e_{i}}^{j}=\omega_{z}^{j}+\omega_{z+e_{j}}^{i} \text { for } i, j=1, \ldots, d, i \neq j\right\},
\end{aligned}
$$

where $e_{i}, i=1, \ldots, d$, denote the unit vectors in $\mathbb{Z}^{d}$. The edges of $\mathbb{Z}^{d}$ are oriented in the natural way (following the three thumb rule). We should think of $\omega_{z}^{i}$ as the label of the edge between $z$ and $z+e_{i}$. The set $\Omega$ should be interpreted as follows: for two vertices $z$ and $z^{\prime}$, let $\pi$ be an edge-self-avoiding path from $z$ to $z^{\prime}$. Travelling from $z$ to $z^{\prime}$ along $\pi$, we add all labels of edges which we traverse in the positive direction and subtract the labels of the edges which we traverse 
in the negative direction. The property in the definition of $\Omega$ asserts that the outcome $g\left(z, z^{\prime}, \omega\right)$ is independent of the choice of $\pi$, and only depends on $z$ and $z^{\prime}$ (and on $\omega$ of course). We define $f(z, \omega)$ to be $g(0, z, \omega)$. Then $f$ is a map $\mathbb{Z}^{d} \times \Omega \rightarrow \mathbb{Z}^{d}$ and if $\phi: \mathbb{Z}^{d} \times \Omega \rightarrow \Omega$ is the group action given by the coordinate shift, then $f$ satisfies the cocycle identity

$$
f\left(z+z^{\prime}, \omega\right)=f(z, \omega)+f\left(z^{\prime}, \phi_{z}(\omega)\right) .
$$

The cocycle $f$ plays the role of the position of the random walk in the onedimensional case, and the labels of the edges play the role of the increments. Let $\mu$ be a $\phi$-invariant ergodic probability measure on $\Omega$ (on the natural $\sigma$ algebra) with the property that the edge labels are uniformly bounded. Henceforth, we will always assume that $\mu$ has this property.

Let $F$ be a finite set containing at least two elements, and consider a $\mathbb{Z}^{d}$. action $\psi$ on $X=F^{\mathbb{Z}^{d}}$, together with an $\psi$-invariant, ergodic measure $\rho$ on $X$. The cocycle $f$ induces a $(\mu \times \rho)$-invariant $\mathbb{Z}^{d}$-action (known as a skew product) $\Phi: \mathbb{Z}^{d} \times \Omega \times X \rightarrow \Omega \times X$ as follows:

$$
\Phi_{z}(\omega, x)=\left(\phi_{z}(\omega), \psi_{f(z, \omega)}(x)\right) .
$$

We continue with the definition of random entropy. We write $h_{m}(\xi)$ for the usual ergodic theoretical entropy with measure $m$ and $\mathbb{Z}^{d}$-action $\xi$.

DEFINITION 2.1. The random entropy $E_{\rho}(\mu)$ is defined as

$$
E_{\rho}(\mu)=h_{\mu \times \rho}(\Phi)-h_{\mu}(\phi)
$$

Note that this is just the fibre entropy of the skew product (see [5]). The above random entropy was studied and explicitly calculated in [1].

As mentioned before, a one-dimensional cocycle is just an ordinary random walk. If this random walk is simple, that is, if $\mu$ is a product measure on $\{1,-1\}^{\mathbb{Z}}$ with the marginals equal to 1 with probability $p$, then the random entropy can be computed and turns out to be equal to $|2 p-1| h_{\rho}(\psi)$ (see $[1,3]$ ). (Compare this with the forthcoming Theorems 2.3 and 3.1.)

Finally, we need to define the notions of recurrence and transience of a cocycle.

DefinItIon 2.2. The cocycle $f$ (or the measure $\mu$ ) is said to be recurrent if

$$
\mu\left(\omega ; f(z, \omega)=0 \text { for infinitely many } z \in \mathbb{Z}^{d}\right)=1 .
$$

The cocycle $f$ (or the measure $\mu$ ) is said to be transient if, for all $z^{\prime} \in \mathbb{Z}^{d}$,

$$
\mu\left(\omega ; f(z, \omega)=f\left(z^{\prime}, \omega\right) \text { for infinitely many } z \in \mathbb{Z}^{d}\right)=0 .
$$


In words, recurrence means infinitely many "visits" to the origin a.s., and transience means that each image vector is attained only finitely many times a.s. It does not follow from the definitions that any given cocycle is either recurrent or transient, though we will now see that this is the case nevertheless.

THEOREM 2.3. Suppose that $0<h_{\rho}(\psi)<\infty$.

(1) If $E_{\rho}(\mu)>0$, then $\mu$ is transient.

(2) If $E_{\rho}(\mu)=0$, then $\mu$ is recurrent.

COROLlary 2.4 (a Polya dichotomy). Any measure $\mu$ (which concentrates on configurations with uniformly bounded edge labels) on $\Omega$ is either recurrent or transient.

Proof. Given a measure $\mu$ on $\Omega$, take a measure $\rho$ on $X$ with finite positive entropy and apply Theorem 2.3.

3. Proof of recurrence. For ease of notation and description, we will stick to the two-dimensional case. Everything we say goes through in all dimensions. Before we start proving anything, we mention at this point that we will go back and forth between probabilistic language and ergodic-theoretical language, depending on which is more suitable for the current purpose. So, for instance, we will use the phrases "one-dimensional cocycle" and "random walk" interchangeably. Also, sometimes we behave like probabilists and do not write the dependence on $\omega$, but occasionally it is convenient to stress on this dependence.

We define horizontal and vertical limits, writing $f=\left(f_{1}, f_{2}\right)$, as follows:

$$
\begin{array}{lll}
h_{1}(k)=\lim _{n \rightarrow \infty} \frac{f_{1}(n, k)-f_{1}(0, k)}{n}, & h_{2}(k)=\lim _{n \rightarrow \infty} \frac{f_{2}(n, k)-f_{2}(0, k)}{n}, \\
v_{1}(k)=\lim _{n \rightarrow \infty} \frac{f_{1}(k, n)-f_{1}(k, 0)}{n}, & v_{2}(k)=\lim _{n \rightarrow \infty} \frac{f_{2}(k, n)-f_{2}(k, 0)}{n} .
\end{array}
$$

All these limits exist $\mu$-a.e. by stationarity. We first claim that $h_{1}(k)$ is independent of $k$ and similarly for the other quantities. To see this, we write $X_{n}$ for $f_{1}(n, k)-f_{1}(0, k)$ and $Y_{n}$ for $f_{1}(n, k+1)-f_{1}(0, k+1)$. We have that $\left|X_{n}-Y_{n}\right| \leq K$ for some uniform $K>0$. Hence,

$$
E\left(\left|\frac{X_{n}}{n}-\frac{Y_{n}}{n}\right|\right) \rightarrow 0
$$

for $n \rightarrow \infty$ and it follows from Markov's inequality that $\left|X_{n} / n-Y_{n} / n\right|$ converges to 0 in probability. Hence the a.e. limit (which is known to exist) has to be 0 as well. This proves the claim.

It follows that $h_{1}(k)$ is invariant under both horizontal and vertical translations and hence it is $\mu$-a.e. constant. Similar statements are valid for the other 
quantities. Therefore, it makes sense to define $h_{1}=h_{1}(k), h_{2}=h_{2}(k), v_{1}=$ $v_{1}(k)$, and $v_{2}=v_{2}(k)$. We write $h=\left(h_{1}, h_{2}\right)$ and $v=\left(v_{1}, v_{2}\right)$. The following result is taken from [1] and gives an explicit formula for the random entropy.

THEOREM 3.1. One has

$$
E_{\rho}(\mu)=|\operatorname{det}(h, v)| h_{\rho}(\psi)
$$

The following result shows that we have convergence in measure to an explicit limit for the values of the cocycle in any given direction. In the remainder of this paper, the quotient $1 /(1+\infty)$ is interpreted as 0 and $\infty /(1+\infty)$ as 1 .

LEMMA 3.2. Let $\left\{\left(k_{n}, m_{n}\right)\right\}$ be a sequence of vectors in $\mathbb{Z}^{2}$.

(i) Suppose that $\left(k_{n}, m_{n}\right) \rightarrow\left(c_{1} \cdot \infty, c_{2} \cdot \infty\right)$ for some $c_{1}, c_{2} \in\{1,-1\}$ and in addition that $m_{n} / k_{n} \rightarrow \alpha \in[-\infty, \infty]$. Then

$$
\frac{f\left(k_{n}, m_{n}\right)}{\left|k_{n}\right|+\left|m_{n}\right|} \rightarrow \frac{c_{1}}{1+|\alpha|} h+\frac{c_{2}|\alpha|}{1+|\alpha|} v
$$

in $\mu$ measure as $n \rightarrow \infty$.

(ii) Suppose that $\left\{k_{n}\right\}$ is bounded and $m_{n} \rightarrow c_{3} \cdot \infty$ for some $c_{3} \in\{1,-1\}$. Then

$$
\frac{f\left(k_{n}, m_{n}\right)}{\left|k_{n}\right|+\left|m_{n}\right|} \longrightarrow c_{3} v
$$

in $\mu$ measure as $n \rightarrow \infty$.

(iii) Suppose that $\left\{m_{n}\right\}$ is bounded and $k_{n} \rightarrow c_{4} \cdot \infty$ for some $c_{4} \in\{1,-1\}$. Then

$$
\frac{f\left(k_{n}, m_{n}\right)}{\left|k_{n}\right|+\left|m_{n}\right|} \longrightarrow c_{4} h
$$

in $\mu$ measure as $n \rightarrow \infty$.

Proof. For (i), we will only prove the case $c_{1}=c_{2}=1$ (and hence $\alpha \geq 0$ ) since the proofs of the other cases are all similar. Let $\epsilon>0$, choose $\epsilon_{1}>0$ so that

$$
\epsilon_{1}\left(\frac{1}{1+\alpha}+\epsilon_{1}\right)+|h| \epsilon_{1}+\epsilon_{1}\left(\frac{\alpha}{1+\alpha}+\epsilon_{1}\right)+|v| \epsilon_{1}<\epsilon
$$

(The reason for this complicated expression will become apparent soon.) Let

$$
\begin{aligned}
& A\left(n, \epsilon_{1}\right)=\left\{\omega ;\left|\frac{f(n, 0, w)}{n}-h\right|<\epsilon_{1}\right\}, \\
& B\left(n, \epsilon_{1}\right)=\left\{\omega ;\left|\frac{f(0, n, w)}{n}-v\right|<\epsilon_{1}\right\} .
\end{aligned}
$$


Using the convergence in measure (we have at this point, in fact, a.s. convergence), there exists $N$ sufficiently large so that, for all $n>N$, one has

(a) $\mu\left(A\left(k_{n}, \epsilon_{1}\right)\right)>1-\epsilon$,

(b) $\mu\left(B\left(m_{n}, \epsilon_{1}\right)\right)>1-\epsilon$,

(c) $\left|1 /\left(1+m_{n} / k_{n}\right)-1 /(1+\alpha)\right|<\epsilon_{1}$,

(d) $\left|\left(m_{n} / k_{n}\right) /\left(1+m_{n} / k_{n}\right)-\alpha /(1+\alpha)\right|<\epsilon_{1}$

hold. Since $\mu$ is a translation invariant measure, for $n>N$, we have

$$
\mu\left(\phi_{(n, 0)} B\left(m_{n}, \epsilon_{1}\right)\right)>1-\epsilon,
$$

and hence

$$
\mu\left(A\left(k_{n}, \epsilon_{1}\right) \cap \phi_{(n, 0)} B\left(m_{n}, \epsilon_{1}\right)\right)>1-2 \epsilon .
$$

Now, for $\omega \in A\left(k_{n}, \epsilon_{1}\right) \cap \phi_{(n, 0)} B\left(m_{n}, \epsilon_{1}\right)$, we have

$$
\begin{aligned}
& \left|\frac{f\left(k_{n}, m_{n}, \omega\right)}{k_{n}+m_{n}}-\frac{1}{1+\alpha} h-\frac{\alpha}{1+\alpha} v\right| \\
& \quad \leq\left|\frac{f\left(k_{n}, 0, \omega\right)}{k_{n}\left(1+m_{n} / k_{n}\right)}-\frac{1}{1+m_{n} / k_{n}} h\right|+\left|\frac{1}{1+m_{n} / k_{n}}-\frac{1}{1+\alpha}\right||h| \\
& \quad+\left|\frac{f\left(0, m_{n}, \phi_{\left(k_{n}, 0\right)} \omega\right)}{m_{n}\left(1+k_{n} / m_{n}\right)}-\frac{1}{1+k_{n} / m_{n}} v\right|+\left|\frac{m_{n} / k_{n}}{1+m_{n} / k_{n}}-\frac{\alpha}{1+\alpha}\right||v| \\
& \quad<\epsilon_{1}\left(\frac{1}{1+\alpha}+\epsilon_{1}\right)+|h| \epsilon_{1}+\epsilon_{1}\left(\frac{\alpha}{1+\alpha}+\epsilon_{1}\right)+|v| \epsilon_{1}<\epsilon
\end{aligned}
$$

where the last inequality follows from the choice of $\epsilon_{1}$.

This implies that for each $n>N$, the set $A\left(k_{n}, \epsilon_{1}\right) \cap \phi_{(n, 0)} B\left(m_{n}, \epsilon_{1}\right)$ is contained in the set

$$
\left\{\omega ;\left|\frac{f\left(k_{n}, m_{n}, \omega\right)}{k_{n}+m_{n}}-\frac{1}{1+\alpha} h-\frac{\alpha}{1+\alpha} v\right|<\epsilon\right\}
$$

Hence,

$$
\mu\left(\left|\frac{f\left(k_{n}, m_{n}, \omega\right)}{k_{n}+m_{n}}-\frac{1}{1+\alpha} h-\frac{\alpha}{1+\alpha} v\right|<\epsilon\right)>1-2 \epsilon
$$

for all $n>N$.

For (ii), recall that $f(0, n) / n$ converges in measure to $v$, it follows that $f(-n, 0) / n$ converges in measure to $-v$. Now we can write

$$
\frac{f\left(k_{n}, m_{n}\right)}{\left|k_{n}\right|+\left|m_{n}\right|}=\frac{f\left(0, m_{n}\right)}{\left|m_{n}\right|} \cdot \frac{\left|m_{n}\right|}{\left|k_{n}\right|+\left|m_{n}\right|}+\frac{f\left(k_{n}, 0, \phi_{\left(0, m_{n}\right)} \omega\right)}{\left|k_{n}\right|+\left|m_{n}\right|} \text {. }
$$


The first term converges to $c_{3} v$ and the second term goes to 0 in probability since $\left\{k_{n}\right\}$ is bounded, using the stationarity of $\mu$. The proof of (iii) is similar and is omitted.

We next prove the second part of Theorem 2.3. First suppose that $E_{\rho}(\mu)=0$. There are two possibilities: (i) either $h$ or $v$ is the zero vector, or (ii) $h$ and $v$ are linearly dependent. If $h$ or $v$ is zero, say $h$, then it follows from Lemma 3.2 that $k_{1}$ defined as

$$
k_{1}(n)=f(n, 0)
$$

has the property that

$$
\frac{k_{1}(n)}{n} \longrightarrow 0
$$

in $\mu$ measure as $n \rightarrow \infty$. Since $k_{1}$ is a random walk with stationary increments, it is well known that (see $[2,4]$ ) this implies that $k_{1}$ is recurrent which in turn implies that $f$ is recurrent.

Next we assume that $h$ and $v$ are nonzero. Then $v=\gamma h$ for some $0 \neq \gamma \in \mathbb{R}$. If $\gamma=p / q \in \mathbb{Q}$, then $k_{2}$ defined as

$$
k_{2}(n)=f(n p,-n q)
$$

is a stationary random walk which, according to Lemma 3.2, satisfies

$$
\frac{k_{2}(n)}{n} \longrightarrow 0
$$

in $\mu$ measure as $n \rightarrow \infty$. As in the previous case, it follows that $k_{2}$ is recurrent and so $f$ is recurrent as well.

Finally suppose that $\gamma$ is irrational and let $\beta=-1 / \gamma$. We generalize the proof of the above cases. That is, we want to pick lattice points close to the line $y=\beta x$ (which is the "recurrence direction") in such a way that the cocycle $f$ evaluated at these lattice points gives a recurrent one-dimensional random walk with stationary increments. This will be possible if we enlarged our probability space. The idea is to move the line $y=\beta x$ by a random uniform distance $\delta \in[0,1]$ in the vertical direction, and on each vertical $x=n$ line, we pick the lattice point closest to the intersection of $y=\beta x+\delta$ with the line $x=n$. The values of the cocycle $f$ evaluated at these points will now be shown to be a random walk with stationary increments. In order to do this completely rigorously, it seems easier to adapt the cocycle language rather than the probabilistic language.

Consider the space $[0,1] \times \Omega$ with the product $\sigma$-algebra and product measure $P \times \mu$, where on $[0,1]$ we have the usual Borel $\sigma$-algebra with $P$ Lebesgue measure. Define $U:[0,1] \times \Omega \rightarrow[0,1] \times \Omega$ by

$$
U(\delta, \omega)=\left((\delta+\beta) \bmod 1, \phi_{(1,\lfloor\delta+\beta\rfloor)} \omega\right) .
$$


Then $U$ is clearly $P \times \mu$ invariant and

$$
U^{n}(\delta, \omega)=\left((\delta+n \beta) \bmod 1, \phi_{(n,\lfloor\delta+n \beta\rfloor)} \omega\right) .
$$

Define $g:[0,1] \times \Omega \rightarrow \mathbb{Z}^{2}$ by

$$
g(\delta, \omega)=f(1,\lfloor\delta+\beta\rfloor, \omega)
$$

and $k: \mathbb{Z} \times[0,1] \times \Omega \rightarrow \mathbb{Z}^{2}$ by

$$
k(n, \delta, \omega)=\sum_{i=0}^{n-1} g\left(U^{i}(\delta, \omega)\right)=f(n,\lfloor\delta+n \beta\rfloor, \omega)
$$

Then $k$ is a cocycle for the $\mathbb{Z}$-action generated by $U$. Since $\lim _{n \rightarrow \infty}\lfloor\delta+n \beta\rfloor / n=$ $\beta, v=\alpha$, and $u=-(1 / \beta) v$, it follows from Lemma 3.2 that, for each $\delta \in[0,1]$,

$$
\frac{f(n,\lfloor\delta+n \beta\rfloor)}{n+|\lfloor\delta+n \beta\rfloor|} \rightarrow \frac{1}{1+|\beta|} h+\frac{\beta}{1+|\beta|} v=0
$$

in $\mu$ measure as $n \rightarrow \infty$. Since $n /(n+|\lfloor\delta+n \beta\rfloor|) \rightarrow 1 /(1+|\beta|)$, this also implies that

$$
\frac{f(n,\lfloor\delta+n \beta\rfloor)}{n} \rightarrow 0
$$

in $\mu$ measure as $n \rightarrow \infty$. We claim that $k(n, \cdot, \cdot) / n \rightarrow 0$ in $P \times \mu$ measure as $n \rightarrow \infty$. To see this, let $\epsilon>0$. According to (3.24), for each $\delta \in[0,1]$, there exists $N_{\delta}$ such that, for all $n \geq N_{\delta}$,

$$
\mu\left(\left|\frac{f(n,\lfloor\delta+n \beta\rfloor, \omega)}{n}\right|<\epsilon\right)>\sqrt{1-\epsilon} .
$$

Also, there exists a constant $M$ such that

$$
P\left(\left\{\delta ; N_{\delta} \leq M\right\}\right)>\sqrt{1-\epsilon} .
$$

Let

$$
\begin{gathered}
C(n, \delta)=\left\{\omega ;\left|\frac{f(n,\lfloor\delta+n \beta\rfloor, \omega)}{n}\right|<\epsilon\right\}, \\
D=\left\{\delta ; N_{\delta} \leq M\right\} .
\end{gathered}
$$


For $n \geq M$, we have

$$
\begin{aligned}
(P \times & \mu)\left(\left\{(\delta, \omega) ;\left|\frac{k(n, \delta, \omega)}{n}\right|<\epsilon\right\}\right) \\
= & (P \times \mu)\left(\left\{(\delta, \omega) ;\left|\frac{f(n,\lfloor\delta+n \beta\rfloor, \omega)}{n}\right|<\epsilon\right\}\right) \\
= & (P \times \mu)(\{(\delta, \omega) ; \omega \in C(n, \delta)\}) \\
= & \int_{0}^{1} \mu(C(n, \delta)) d P(\delta) \\
& \geq \int_{D} \mu(C(n, \delta)) d P(\delta) \geq 1-\epsilon .
\end{aligned}
$$

This proves the claim. Since $k$ is a cocycle for the $\mathbb{Z}$-action generated by $U$, it follows as before that $k$ is recurrent, that is,

$$
(P \times \mu)(\{(\delta, \omega) ; k(n, \delta, \omega)=0 \text { for infinitely many } n \in \mathbb{Z}\})=1 .
$$

Projecting on the second coordinate yields that, for a.e. $\delta$, we have

$$
\mu(\{\omega ; f(n,\lfloor\delta+n \beta\rfloor, \omega)=0 \text { for infinitely many } n \in \mathbb{Z}\})=1 .
$$

In fact, we only need one $\delta$ with this property. Anyway, it follows that $f$ is recurrent.

For future use, we state the following consequence of the previous construction.

LEMMA 3.3. For any $\alpha \in[-\infty, \infty]$, (random) vertices $\left(y_{0}, y_{1}, y_{2}, \ldots\right)$ can be constructed such that $\left(f\left(y_{0}\right), f\left(y_{1}\right), \ldots\right)$ forms a random walk with stationary increments defined on the probability space $[0,1] \times \Omega$ such that

(i) $y_{n, 2} / y_{n, 1} \rightarrow \alpha$ (where $y_{n}=\left(y_{n, 1}, y_{n, 2}\right)$ ),

(ii) $\left|y_{n+1}-y_{n}\right|$ is uniformly bounded (where the bound depends on $\alpha$ ) for $n \geq 0$.

Proof. When $h$ (say) is zero, take $y_{n}=(n, 0)$; when $v=\gamma h$ for $\gamma=p / q \in$ $\mathbb{Q}$, take $y_{n}=(n p,-n q)$; and when $v=\gamma h$ for irrational $\gamma$, take $y_{n}=\lfloor\delta+n \beta\rfloor$ as in the above construction.

4. Proof of transience. We start with a strengthening of Lemma 3.2. We will need convergence as in Lemma 3.2 along a random sequence $\left(k_{n}, m_{n}\right)$. The choice of this sequence will depend on the realisation, so some care is needed. It turns out that it is easier to work with a.s. convergence in this context. It does not suffice, however, to just change the mode of convergence in Lemma 3.2 to a.s. convergence. The reason for this is that each sequence $\left(k_{n}, m_{n}\right)$ has an exceptional set of measure zero where convergence does not take place. We need to guarantee that the realisation we see is not in the exceptional set of the random sequence $\left(k_{n}, m_{n}\right)$ which, after all, depends on this very configuration. That is, we need some uniformity in our estimates. 
LemmA 4.1. Let $\alpha \in(-\infty, \infty)$. Then for any $\epsilon>0$, there a.s. exist $N_{\epsilon}>0$ and $\delta_{\epsilon}>0$ such that whenever $m_{n}, k_{n}>N_{\epsilon}$ and $\left|m_{n} / k_{n}-\alpha\right|<\delta_{\epsilon}$,

$$
\left|\frac{f\left(k_{n}, m_{n}\right)}{\left|k_{n}\right|+\left|m_{n}\right|}-\frac{1}{1+|\alpha|} h-\frac{|\alpha|}{1+|\alpha|} v\right|<\epsilon
$$

When $\alpha= \pm \infty, \delta_{\epsilon}$ should be replaced by a constant $M_{\epsilon}$ and the condition $\left|m_{n} / k_{n}-\alpha\right|<\delta_{\epsilon}$ should be replaced by $\left|m_{n} / k_{n}\right|>M_{\epsilon}$. Moreover, similar statements are valid for all other cases of Lemma 3.2.

PROOF. For the purists among us, first note that "a.s." in the statement of the lemma refers to $\mu$. In the proof to follow, a.s. refers to $(P \times \mu)$ as defined in Section 3. The result then follows by projecting on the second coordinate.

For the given $\alpha$, we choose the sequence of points $\left(y_{n}\right)$ dictated by Lemma 3.3. We write $x_{n}=\left(k_{n}, m_{n}\right)$ and write $y_{j(n)}$ for the (or a) vertex among $\left(y_{0}, y_{1}, \ldots\right)$ which is closest to $x_{n}$. We then have

$$
\frac{f\left(x_{n}\right)}{\left\|x_{n}\right\|}=\left(\frac{f\left(y_{j(n)}\right)}{\left\|y_{j(n)}\right\|}+\frac{f\left(x_{n}\right)-f\left(y_{j(n)}\right)}{\left\|y_{j(n)}\right\|}\right) \frac{\left\|y_{j(n)}\right\|}{\left\|x_{n}\right\|}
$$

The ergodic theorem tells us that $f\left(y_{n}\right) /\left\|y_{n}\right\|$ converges a.s., and it then follows from the corresponding convergence in measure in Lemma 3.2 that this a.s. limit must be the same limit as in Lemma 3.2. Therefore, if $k_{n}$ and $m_{n}$ are large enough and $\left|m_{n} / k_{n}-\alpha\right|$ is small enough, then $j(n)$ is large and therefore $f\left(y_{j(n)}\right) /\left\|y_{j(n)}\right\|$ is close to the correct limit in Lemma 3.2. At the same time, the term $\left\|y_{j(n)}\right\| /\left\|x_{n}\right\|$ is close to 1 by construction. Finally, the norm of the vector $\left(f\left(x_{n}\right)-f\left(y_{j(n)}\right)\right) /\left\|y_{j(n)}\right\|$ is bounded above by

$$
\frac{M\left\|x_{n}-y_{j(n)}\right\|}{\left\|y_{j(n)}\right\|}
$$

where $M$ is the uniform upper bound on the norm of the edge labels. This last expression is close to 0 when $k_{n}$ and $m_{n}$ are large and $\left|m_{n} / k_{n}-\alpha\right|$ is small.

For our next lemma, we need some additional notation. For each integer $n$, define half-planes as follows:

$$
\begin{aligned}
& H_{1}(n)=\left\{(x, y) \in \mathbb{R}^{2} ; y \leq n\right\}, \\
& H_{2}(n)=\left\{(x, y) \in \mathbb{R}^{2} ; x \leq n\right\}, \\
& H_{3}(n)=\left\{(x, y) \in \mathbb{R}^{2} ; y \geq n\right\}, \\
& H_{4}(n)=\left\{(x, y) \in \mathbb{R}^{2} ; x \geq n\right\} .
\end{aligned}
$$


LEMmA 4.2. Suppose that $|\operatorname{det}(h, v)|>0$. Then there exist random variables $N_{1}, N_{2}, N_{3}$, and $N_{4}$, taking values in the positive integers such that

$$
\begin{array}{ll}
f(0, n) \notin f\left(H_{1}(0)\right) & \forall n \geq N_{1}, \\
f(n, 0) \notin f\left(H_{2}(0)\right) & \forall n \geq N_{2}, \\
f(0, n) \notin f\left(H_{3}(0)\right) & \forall n \leq-N_{3}, \\
f(n, 0) \notin f\left(H_{4}(0)\right) & \forall n \leq-N_{4} .
\end{array}
$$

Proof. We will only prove the existence of $N_{1}$ since the other cases are proved similarly.

We call $\omega \in \Omega$ very bad if there exist a sequence $\left\{z_{k}\right\}$ in $H_{1}(0)$ and an infinite sequence $0<n_{1}<n_{2}<\cdots$ of positive integers such that $f\left(z_{k}, \omega\right)=$ $f\left(0, n_{k}, \omega\right)$ for all $k \geq 1$. Let

$$
B=\{\omega ; \omega \text { is very bad }\} .
$$

It suffices to show that $\mu(B)=0$. A problem here is that $B$ is not clearly translation invariant. To overcome this difficulty, we enlarge the set $B$ as to get an invariant set.

We call $\omega$ bad if for some $m$ and $\ell$, there exist an infinite set of distinct points $W=\left\{w_{1}, w_{2}, \ldots\right\} \in[-m, m] \times\{0,1,2, \ldots\}$ and a set $\mathrm{Z}=\left\{z_{1}, z_{2}, \ldots\right\}$ of lattice points in $H_{1}(\ell)$ such that $f\left(z_{k}, \omega\right)=f\left(w_{k}, \omega\right)$. It is clear that the set

$$
A=\{\omega ; \omega \text { is bad }\}
$$

is translation invariant and hence by ergodicity $\mu(A)$ is either 0 or 1 . Furthermore, we have $B \subseteq A$ so that it suffices to prove that $\mu(A)=0$. We now assume that $\mu(A)=1$ and show that we get a contradiction.

It follows from Lemma 4.1 that

$$
\frac{f\left(w_{k}\right)}{\left\|w_{k}\right\|} \longrightarrow v
$$

a.s., where $\|\cdot\|$ denotes $L_{1}$ distance.

The first thing is to rule out the possibility of the set $Z$ being finite. This is not hard. We can write

$$
\frac{f\left(w_{k}\right)}{\left\|w_{k}\right\|}=\frac{f\left(z_{k}\right)}{\left\|z_{k}\right\|} \cdot \frac{\left\|z_{k}\right\|}{\left\|w_{k}\right\|}
$$

The left-hand side converges a.s. to $v$ which is not the zero vector by assumption. On the event that $Z$ is bounded, the right-hand side converges a.s. to the zero vector. Therefore, $Z$ is unbounded a.s.

Next we let $a\left(z_{k}\right)$ be the angle that the vector $z_{k}$ makes with the positive $x$-axis, measured counterclockwise. We define $\Theta=\Theta(\omega)$ as the (random) set of limit points of $\left\{a\left(z_{k}\right)\right\}$. Since the $z_{k}$ 's are all in $H_{1}(\ell)$ for some $\ell$, we have 
that $\Theta$ is nonempty and satisfies $\Theta \subseteq[\pi, 2 \pi]$. Since $\Theta$ is also closed, we can define

$$
\bar{\theta}=\sup \Theta .
$$

Note that $\Theta$ is clearly translation invariant, and therefore $\bar{\theta}$ is an almost sure constant. Now choose a subsequence $\left(z_{k_{1}}, z_{k_{2}}, \ldots\right)$ such that

$$
\frac{z_{k_{n}, 2}}{z_{k_{n}, 1}} \longrightarrow \tan \bar{\theta}
$$

Note that this subsequence is random. Now choose a sequence $\left(\epsilon_{m}\right)$ converging to zero. Using Lemma 4.1, we see that for fixed $m$, a.s. for all $n$ large enough, and for the appropriate $\beta_{1}$ and $\beta_{2}$,

$$
\left|\frac{f\left(z_{k_{n}}\right)}{\left\|z_{k_{n}}\right\|}-\beta_{1} v-\beta_{2} h\right|<\epsilon_{m} .
$$

The exceptional set depends on $m$ but the intersection of these sets (countably many) still has full measure. On this intersection, we get a.s. convergence.

We claim that $\beta_{1} \leq 0$. To see this, note that in Lemma 3.2, either case (i) with $c_{2}=-1$, case (ii) with $c_{3}=-1$, or case (iii) without condition on $c_{4}$ applies. In all these cases, the coefficient of $v$ in the limit is at most 0 .

Using (4.9) again, with $k_{n}$ replacing $k$, we see that the left-hand side still converges a.s. to $v$. According to Lemma 4.1, the first term on the right-hand side converges a.s. to a different vector, which is either linearly independent of $v$ or a nonpositive multiple of $v$. (Here we have used the fact that $h$ and $v$ are linearly independent and the fact that $\beta_{1} \leq 0$.) The second term is, for all $n$, a (random) positive number, and hence we have arrived at a contradiction.

Finally, we show that $\mu$ is transient when $E_{\rho}(\mu)>0$. For this, we define the following stochastic processes:

$$
\begin{aligned}
& Y_{n}^{1}(k)=\min \left\{N \geq 0 ; f(k,-n+\ell) \notin f\left(H_{1}(-n)\right) \forall \ell \geq N\right\}, \\
& Y_{n}^{2}(k)=\min \left\{N \geq 0 ; f(-n+\ell, k) \notin f\left(H_{2}(-n)\right) \forall \ell \geq N\right\}, \\
& Y_{n}^{3}(k)=\min \left\{N \geq 0 ; f(k, n-\ell) \notin f\left(H_{3}(n)\right) \forall \ell \geq N\right\}, \\
& Y_{n}^{4}(k)=\min \left\{N \geq 0 ; f(n-\ell, k) \notin f\left(H_{4}(n)\right) \forall \ell \geq N\right\} .
\end{aligned}
$$

The idea behind these definitions is the following: $Y_{n}^{1}(k)$, for instance, is a random variable that indicates how far we need to go into the box $[-n, n]^{2}$ from below in order to make sure that no value in the lower half-plane $H_{1}(n)$ is seen on the vertical line $x=k$ further up.

It follows from Lemma 4.2 that $Y_{n}^{i}(k)$ is well defined and finite a.s. Specialising to $Y_{n}^{1}$, note that $\left(Y_{n}^{1}(0)\right)_{n}$ is a stationary process. Hence there a.s. exists 
a (random) number $n_{1}$ such that $Y_{n_{1}}^{1}(0)<n_{1}$. It follows from the constructions that this implies that, for all $n \geq n_{1}$, we have $Y_{n}^{1}(0)<n$. For the other processes, $Y^{2}, Y^{3}$, and $Y^{4}$, we find numbers $n_{2}, n_{3}$, and $n_{4}$ such that, for all $n \geq n_{i}$, we have $Y_{n}^{i}(0)<n, i=2,3,4$.

Next define the (random) set $A_{n} \subset[-n, n]^{2}$ as all points $\left(z_{1}, z_{2}\right)$ in $[-n, n]^{2}$ with the property that

$$
\begin{aligned}
& -n+Y_{n}^{2}\left(z_{1}\right) \leq z_{1} \leq n-Y_{n}^{4}\left(z_{1}\right), \\
& -n+Y_{n}^{1}\left(z_{2}\right) \leq z_{2} \leq n-Y_{n}^{3}\left(z_{2}\right) .
\end{aligned}
$$

For all $n>\max \left\{n_{1}, n_{2}, n_{3}, n_{4}\right\}$, we have that the origin is contained in the set $A_{n}$. This implies that for these values of $n$, the value $f(0, \omega)=0$ of the cocycle taken at the origin is not taken at any point outside $B_{n}$.

It is not hard to adapt this argument to other vertices $z^{\prime}$ as well, and this implies that the cocycle is transient.

\section{REFERENCES}

[1] R. Burton, K. Dajani, and R. Meester, Entropy for random group actions, Ergodic Theory Dynam. Systems 18 (1998), no. 1, 109-124.

[2] F. M. Dekking, On transience and recurrence of generalized random walks, Z. Wahrsch. Verw. Gebiete 61 (1982), no. 4, 459-465.

[3] Y. Kifer, Ergodic Theory of Random Transformations, Progress in Probability and Statistics, vol. 10, Birkhäuser Boston, Massachusetts, 1986.

[4] K. Schmidt, On recurrence, Z. Wahrsch. Verw. Gebiete 68 (1984), no. 1, 75-95.

[5] T. Ward and Q. Zhang, The Abramov-Rokhlin entropy addition formula for amenable group actions, Monatsh. Math. 114 (1992), no. 3-4, 317-329.

Karma Dajani: Department of Mathematics, Faculty of Mathematics and Computer Science, University of Utrecht, P.O. Box 80.010, 3508 TA Utrecht, The Netherlands

E-mail address: dajani@math.uu.n1

Ronald Meester: Department of Mathematics, Faculty of Sciences, Vrije Universiteit, De Boelelaan 1081A, 1081 HV Amsterdam, The Netherlands

E-mail address: rmeester@cs.vu.n1 


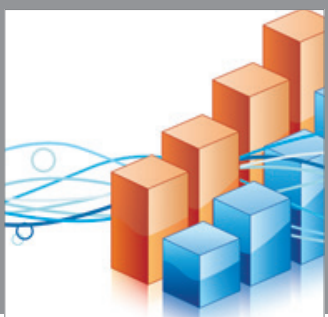

Advances in

Operations Research

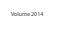

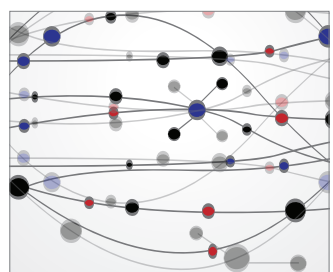

\section{The Scientific} World Journal
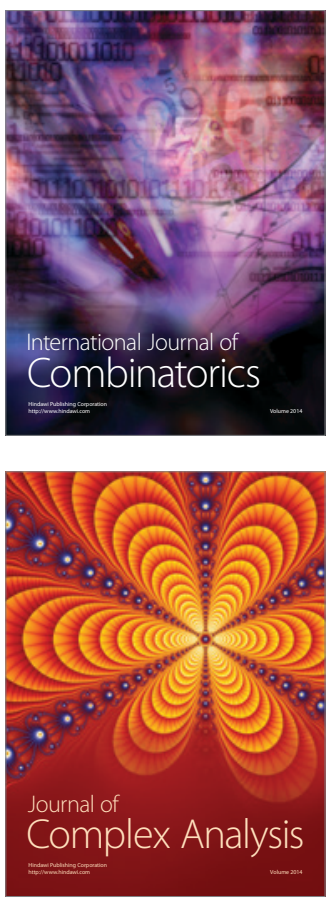

International Journal of

Mathematics and

Mathematical

Sciences
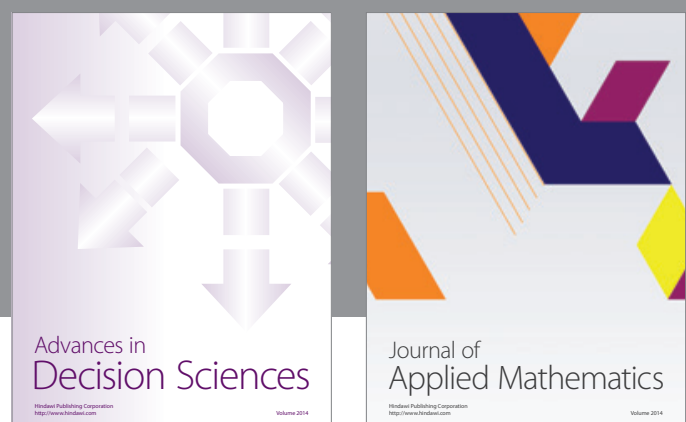

Journal of

Applied Mathematics
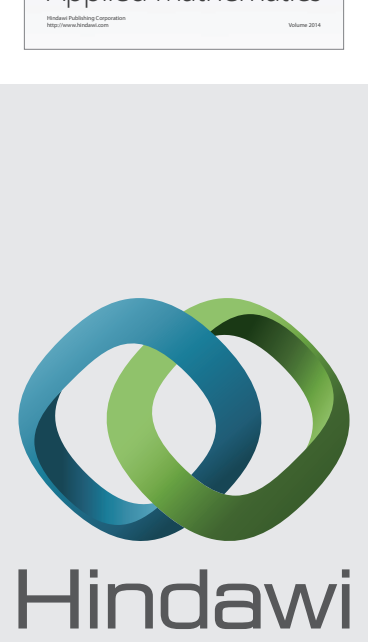

Submit your manuscripts at http://www.hindawi.com
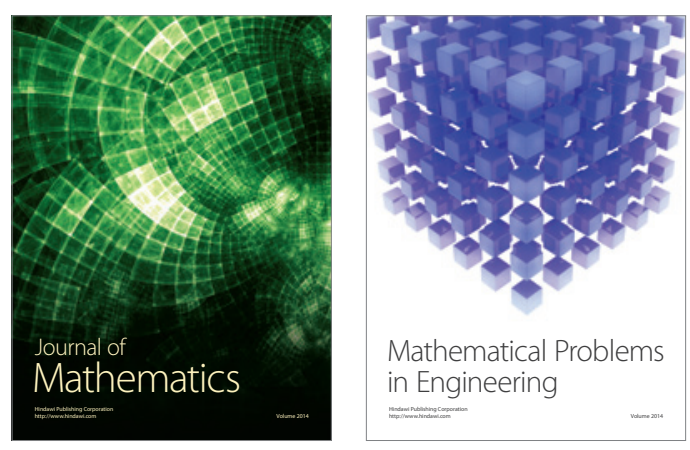

Mathematical Problems in Engineering
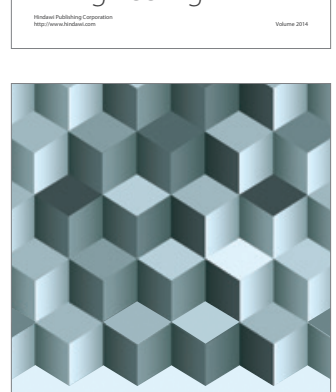

Journal of

Function Spaces
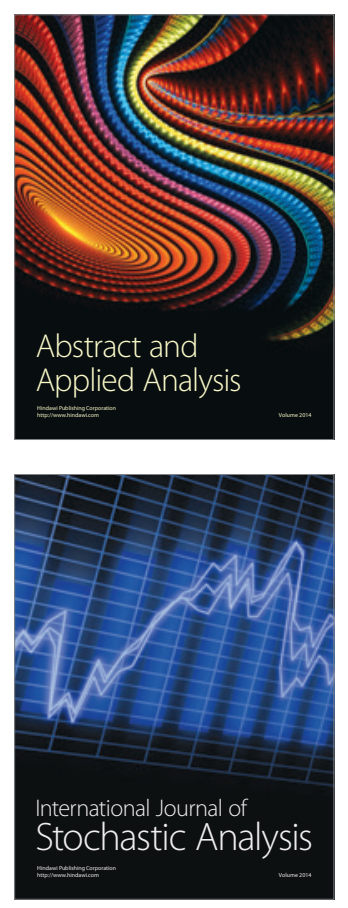

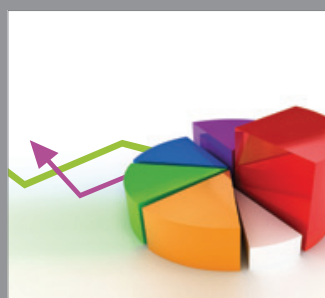

ournal of

Probability and Statistics

Promensencen
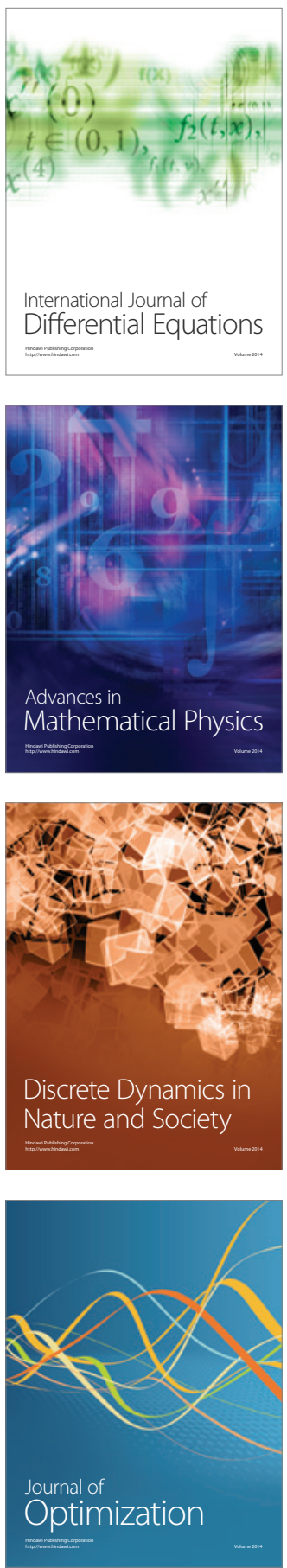